$1-1-2005$

\title{
Doubling the optical efficiency of a chiral liquid crystal laser using a reflector
}

\author{
Ying Zhou \\ University of Central Florida \\ Yuhua Huang \\ University of Central Florida \\ Alexandra Rapaport \\ University of Central Florida \\ Michael Bass \\ University of Central Florida \\ Shin-Tson Wu \\ University of Central Florida
}

Find similar works at: https://stars.library.ucf.edu/facultybib2000

University of Central Florida Libraries http://library.ucf.edu

This Article is brought to you for free and open access by the Faculty Bibliography at STARS. It has been accepted for inclusion in Faculty Bibliography 2000s by an authorized administrator of STARS. For more information, please contactSTARS@ucf.edu.

\section{Recommended Citation}

Zhou, Ying; Huang, Yuhua; Rapaport, Alexandra; Bass, Michael; and Wu, Shin-Tson, "Doubling the optical efficiency of a chiral liquid crystal laser using a reflector" (2005). Faculty Bibliography 2000s. 5857. https://stars.library.ucf.edu/facultybib2000/5857

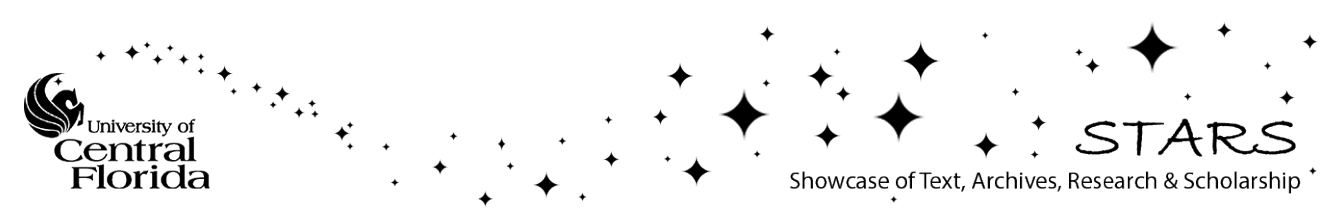




\title{
Doubling the optical efficiency of a chiral liquid crystal laser using a reflector
}

\author{
Ying Zhou, Yuhua Huang, Alexandra Rapaport, Michael Bass, and Shin-Tson Wua) \\ College of Optics and Photonics, University of Central Florida, Orlando, Florida 32816
}

(Received 7 July 2005; accepted 5 October 2005; published online 29 November 2005)

\begin{abstract}
A simple method for doubling the laser emission efficiency of $\mathrm{Nd}$ :yttrium-aluminum-garnet pumped cholesteric liquid crystal (CLC) photonic band edge lasers is demonstrated. By adding a mirror or a CLC reflector behind the dye-doped CLC cell, the lasing intensity is nearly doubled. For the mirror reflector, due to the mixing of right-handed and left-handed circular polarizations, the output laser is basically unpolarized. However, for the CLC reflector, a partially coherent laser can be built in which either right-handed or left-handed circularly polarized laser could dominate the output, depending on the handedness of the cholesteric structure. (C) 2005 American Institute of Physics. [DOI: 10.1063/1.2138353]
\end{abstract}

The dye-doped photonic band edge (PBE) laser represents an emerging class of lasers that do not need an external mirror cavity. ${ }^{1}$ As an example, cholesteric liquid crystal $^{2}$ (CLC) is a promising one-dimensional photonic band gap (PBG) material showing a photonic stop band for circularly polarized light with the same handedness as cholesteric helix. The central wavelength $\left(\lambda_{0}\right)$ and the bandwidth $(\Delta \lambda)$ of the reflection band are determined by $\lambda_{0}=p\langle n\rangle$ and $\Delta \lambda$ $=p \Delta n$, where $\langle n\rangle, \Delta n$, and $p$ represent the average refractive index, birefringence of the liquid crystal host, and intrinsic pitch length, respectively. This self-organized PBG structure opens a way to generate the mirrorless laser action based on the distributed feedback resonance. When triggered by the excitation laser, lasing from the embedded dyes takes place at the photonic band edge because the group velocity near the band edge is substantially reduced and photon dwell time is enhanced correspondingly. Besides CLC, ${ }^{3,4}$ several other liquid crystal hosts, such as ferroelectric liquid crystals, ${ }^{5}$ blue phase, 6 and other materials as well sharing a similar chiral PBG structure as elastomers ${ }^{7}$ and polymers ${ }^{8}$ have been widely investigated. Furthermore, tunable PBE lasing has also been achieved by the control of external stimuli as electrical field, ${ }^{9}$ temperature, or photochemical effect. ${ }^{10-12}$

The optical pumping efficiency of the CLC laser depends on several factors. A typical CLC laser efficiency is $\sim 1 \%-5 \%$ for single output. Several approaches have been proposed for improving the pumping efficiency. For example, cooling the dye-doped CLC device by $10{ }^{\circ} \mathrm{C}$ would lead to an order parameter increase which, in turn, results in a $36 \%$ increase of the slope efficiency. ${ }^{13}$ From a material viewpoint, low molar mass liquid crystals with a high birefringence, extraordinary refractive index, and order parameter of emission transition dipole moment tend to enhance the lasing efficiency. ${ }^{14}$ In addition, low-energy edge lasing is more favorable for high efficiency and low threshold energy due to the highly ordered emission transition moment along the molecular directors. ${ }^{15}$

In this letter, we demonstrate a simple reflective scheme for doubling the pumping efficiency from single direction (either forward or backward) of a CLC laser. Since the stimulated emission only gets effective resonance in the di-

\footnotetext{
${ }^{a)}$ Electronic mail: swu@ mail.ucf.edu
}

rection of periodic modulation with the same sense of circular polarization as the sample itself, it could be observed from both sides perpendicular to the sample substrates. However, for practical use, only the laser from one side can be taken full advantages and the laser beam from another side is wasted. We venture to recycle the laser by adding a mirror or a CLC reflector to the backside of the liquid crystal cell. Under the reflective setup and oblique incidence, a quasiunpolarized laser with the doubled total lasing intensity is obtained if a mirror is employed. While for the CLC reflector, lasing from the front side can be also boosted into a partially coherent laser by nearly twice but is still dominated by the same polarization state. The pumping efficiency of the CLC laser is critically dependent on the chosen material, dye concentration, cell gap and uniformity, pump wavelength, band edge location, etc. Hence the observed 1\%-2\% optical efficiency of our sample implies that we are not operating the laser at its optimal condition. Nevertheless, our goal is to demonstrate a simple method for enhancing the laser efficiency. This method is so general that it can be applied to other CLC laser systems equally well.

In experiment, we prepared a right-handed CLC host by mixing a chiral dopant (Merck CB15) in a positive dielectric anisotropic nematic LC host (Merck BL006). The helical twisting power of $\mathrm{CB} 15$ is $7.71 / \mu \mathrm{m}$ wt $\%$ and the doping concentration is $36 \%$. The laser dye 4-(dicyanomethylene)2-methyl-6-(4-dimethlyaminostryl)-4H-pran (Exciton DCM) was dissolved into the cholesteric host at $2 \%$ concentration. The mixture was then capillary filled into a $15 \mu \mathrm{m} \mathrm{LC}$ cell under isotropic phase and then slowly cooled down at $0.5^{\circ} \mathrm{C} / \mathrm{min}$ to form a uniform cholesteric planar (Grandjean) structure. The inner surfaces of the glass substrates were coated with a thin indium-tin-oxide layer and the polyimide layers were rubbed in the antiparallel directions. The pretilt angle is $\sim 3^{\circ}$. Another two $5 \mu \mathrm{m}$ CLC cells, made of BL006 and 34\% CB15, were used as CLC reflector and CLC lefthanded circularly polarized filter (CLC filter), whose reflection band is centered at the lasing wavelength.

Figure 1 shows the experimental setup. As the excitation source, the $Q$-switched Nd:yttrium-aluminum-garnet (YAG) pulsed laser (Continuum, Minilite II) was operated at $\lambda$ $=532 \mathrm{~nm}, 1 \mathrm{~Hz}$ repetition rate, and $6 \mathrm{~ns}$ pulse width. The excitation laser beam was separated by a beam splitter into 


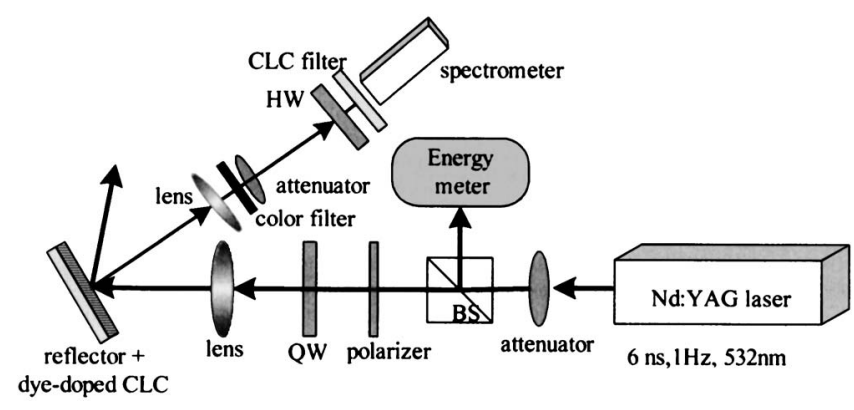

FIG. 1. Experimental setup of a mirrorless CLC laser. BS: beam splitter, QW: quarter-wave plate, and HW: half-wave plate.

two paths. One path was monitored by an energy meter (Ophir, Laserstar) and the other was focused into the lasing cell with $150 \mu \mathrm{m}$ spot diameter. Then a polarizer and a broadband quarter-wave plate were used to change the linearly polarized pumping beam into the left-handed circularly polarized beam. Because the reflection band gap shifts to a shorter wavelength as the incident angle increases, the circularly polarized pumping in the counter handedness from the lasing cell would help reduce the band gap reflection dramatically (if the band gap shift is large enough to cover the pumping wavelength) as compared to the linearly polarized beam. ${ }^{16}$ Pumped at 20 deg oblique incidence, the reflected laser emission from the dye-doped CLC cell was collected in the direction exactly perpendicular to the substrates. The emitted spectrum was measured by a fiber-based spectrometer (Ocean Optics, HR2000). A mirror or a CLC reflector has to be attached tightly to the lasing cell, otherwise, the reflected beam may not coincide with the reflective emission beam. A single CLC filter, or a combination of a CLC filter and a broadband half-wave plate, was placed before the spectrometer to detect the left-handed (LH) or the right-handed (RH) circularly polarized light component, respectively.

Figure 2 plots the reflection band of the CLC lasing cell and the CLC reflector (CLC filter), and the laser emission line centered at $\lambda=635 \mathrm{~nm}$. Lasing occurs at low-energy edge of the band gap where it gains high reflectivity in the distributed feedback cavity. The missing of high-energy band

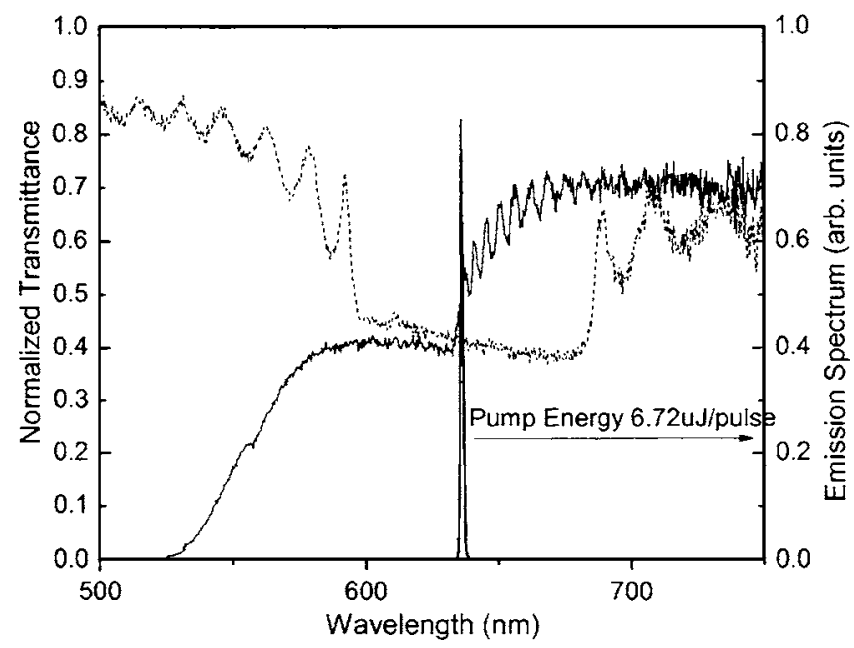

FIG. 2. Transmittance and emission spectra of dye-doped CLC, and transmittance of CLC reflector. Thin solid and dashed lines represent the transmittance of dye-doped CLC cell and CLC reflector, respectively. Thick solid line represents the lasing spectrum under left-handed circularly polarized pumping at $6.72 \mu \mathrm{J} /$ pulse and $20 \mathrm{deg}$ oblique incidence.

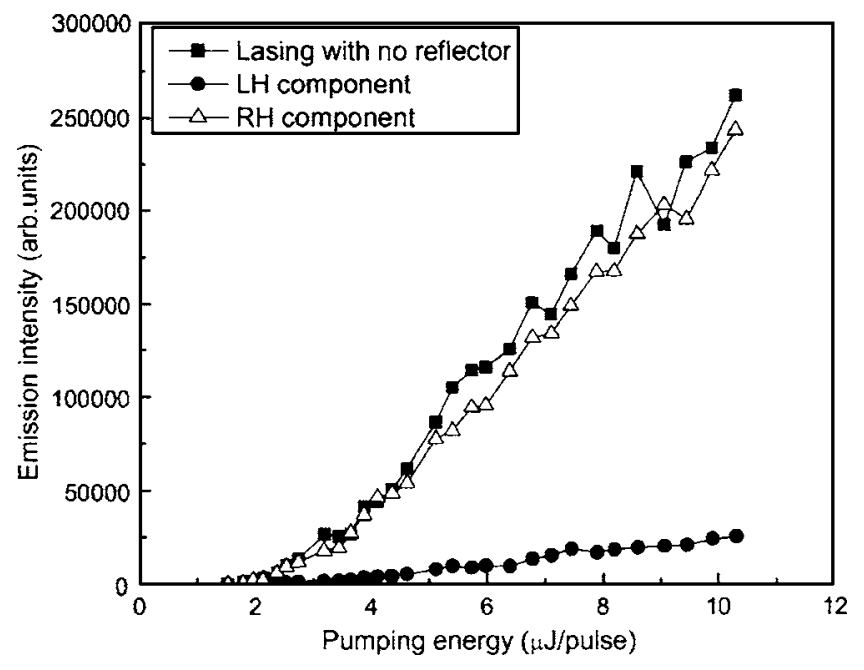

FIG. 3. Input-output relation of a Nd-YAG pumped CLC laser with no reflector. The line with filled squares, open triangles, and filled circles represent the total emission intensity, right-handed, and left-handed circular component intensity, respectively.

edge in CLC lasing cell is due to the DCM dye absorption. The measured full width of half maximum of the narrow, sharp laser line shape is $\sim 0.5 \mathrm{~nm}$ in a stable emission.

Figure 3 depicts the peak laser emission intensity with respect to the excitation energy, i.e., the slope efficiency without reflector attached. The excitation threshold is $\sim 1.3 \mu \mathrm{J} /$ pulse. Above the threshold, the peak intensity increases linearly with the excitation energy except for some fluctuations originated from the pumping laser. The laser emission is mainly right-handed circularly polarized $(\mathrm{RH}$ component), which is in accordance with the CLC helical structure. Only $\sim 10 \%$ of the light is left-handed circularly polarized (LH component), which is believed to result from the imperfection of the CLC planar structure. In the vicinities of the spacer balls, the CLC alignment is disturbed.

The mirror-enhanced pumping efficiency is shown in Fig. 4. Figure 4 compares the optical efficiency of the CLC laser on the total intensity, LH component, and RH compo-

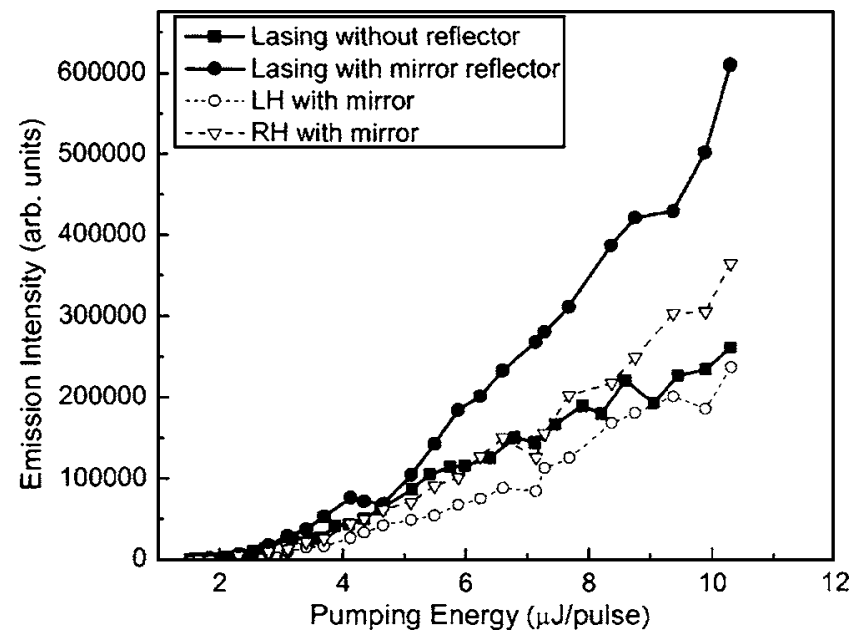

FIG. 4. Pump energy dependent peak intensity of the red laser emission with a mirror reflector. Solid lines with filled circles and filled squares represent the total lasing intensity with and without the reflector, respectively. The dashed lines with open circles and open triangles represent the lefthanded and right-handed circular component intensity with a mirror reflector. 


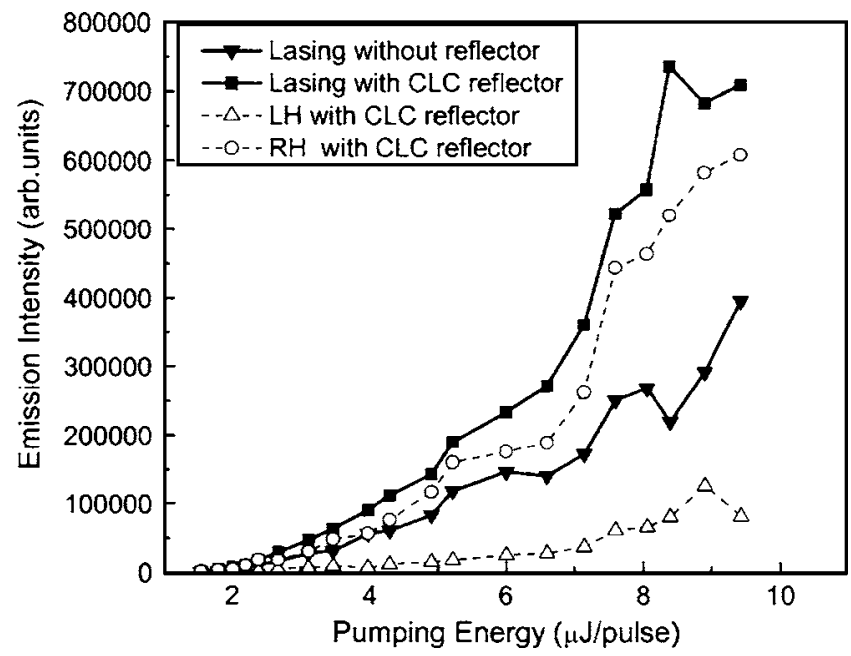

FIG. 5. Pump energy dependent peak intensity of the red laser emission with a CLC reflector. Solid lines with filled squares and filled triangles represent the total lasing intensity with and without a CLC reflector, respectively. The dashed lines with open triangles and open circles represent the left-handed and right-handed circular component intensity with a CLC reflector.

nent when an external mirror is attached. Ideally, the mirror should be deposited in the inner surface of the bottom substrate. The results here demonstrate that the mirror changes the incoming RH laser emission into LH due to the $\pi$ phase change upon reflection. When reflected back into the cell, the LH laser component directly passes through the cell without appreciable polarization deformation and, hence, no resonance is associated. This contributes to the laser intensity enhancement by $\sim 2 \times$ as compared to the scenario with no reflector. Due to interface reflections, the LH component is slightly less than the RH. As a result, the output laser beam becomes quasi-unpolarized based on the incoherent superposition of the LH and RH components. This is equivalently supported by the fact that no distinct linear polarization direction is detected for the output laser. In the far field, the laser beam diverges quickly yet maintains a single spot.

The laser emission enhancement using a CLC reflector, as shown in Fig. 5, is of particular interest. Results show that the total laser intensity is increased by $\sim 2 \times$ and the $R H$ component dominates, which accounts for more than $85 \%$. As stated earlier, the band gap of the CLC reflector covers the lasing wavelength. Unlike a mirror reflector, the laser beam reflected by the CLC reflector preserves its original polarization state, ${ }^{16}$ namely $\mathrm{RH}$. Such a laser beam as edge wavelength and highly reflective polarization state is not expected to pass through the lasing cell. However, a remarkable enhancement is still observed in both total lasing intensity and RH component.

In order to investigate how much $\mathrm{RH}$ laser light at $\lambda$ $=635 \mathrm{~nm}$ could penetrate through the lasing cell itself the following experiment was performed. Two identical lasing cells were cascaded with a color filter sandwiched in between and the first cell was pumped by the frequencydoubled Nd:YAG laser. We found that $\sim 1 / 8$ of the $635 \mathrm{~nm}$, RH laser light produced by the first cell can pass through the second one. Nevertheless further amplification of the reflected beam inside the cavity is believed to originate from the increased cavity length. With a CLC reflector the feed- back length of the cavity is equivalently doubled because the polarization state of the reflected beam has the same handedness as the lasing CLC cell. Moreover, the wavelength is at the band edge which will be facilitated by the polarizationdependent CLC-based distributed feedback cavity. Under the reflective scheme, both gain and loss will be doubled, whose difference will determine how much performance enhancement can be obtained. In a $15 \mu \mathrm{m}$ CLC laser, the imperfection of anisotropic periodicity comes from the peturbation of LC cell's inner spacer balls and the insufficient surface anchoring energy. As the main loss in the cavity, the light scattering from cholesteric defects limits the enhancement factor. The two glass substrates in proximity contact form an isotropic dielectric layer with $2.2 \mathrm{~mm}$ thick. The equivalent optical path difference can be calculated as $\mathrm{OPD}=2 n_{\text {glass }} d_{\text {glass }}=2$ $\times 1.5 \times 2.2=6.6 \mathrm{~mm}$. Since the OPD of the glass substrates is larger than the laser coherence length, the additionally reflected part of the output is, to some extent, incoherent with the initial part. Although the enhancement in laser emission can be obviously observed, the coherence of laser light is somewhat sacrificed, which causes undesirable linewidth broadening of the laser beam. As a result, adding a CLC reflector increases the CLC laser output but its coherence is slightly deteriorated. More detailed theoretical work for further understanding the enhanced $\mathrm{RH}$ output is still under way.

In conclusion, we experimentally demonstrated the DFB based CLC laser enhancement by a mirror and a CLC reflector. For both schemes, the total intensity enhancement is around twice. For the mirror reflector, a quasiunpolarized laser is obtained while for the CLC reflector partially coherent laser is built with single circular polarization state dominant, depending on the CLC structure. This single-side optical efficiency enhancement method can be applied to other CLC laser systems as well.

${ }^{1}$ V. I. Koppa, Z. Q. Zhang, and A. Z. Genack, Prog. Quantum Electron. 27, 369 (2003)

${ }^{2} \mathrm{~S}$. T. Wu and D. K. Yang, Reflective Liquid Crystal Displays (Wiley, New York, 2001).

${ }^{3}$ V. I. Kopp, B. Fan, H. K. M. Vithana, and A. Z. Genack, Opt. Lett. 23, 1707 (1998).

${ }^{4}$ A. Munoz, P. P. Muhoray, and B. Taheri, Opt. Lett. 26, 804 (2001).

${ }^{5}$ M. Ozaki, M. Kasano, T. Kitasho, D. Ganzke, W. Hasse, and K. Y. Oshino, Adv. Mater. (Weinheim, Ger.) 14, 306 (2002).

${ }^{6}$ W. Y. Cao, A. Munoz, P. P. Muhoray, and B. Taheri, Nat. Mater. 1, 111 (2002).

${ }^{7}$ P. A. Bermel and M. Warner, Phys. Rev. E 65, 056614 (2002).

${ }^{8}$ T. Lee, O. Park, H. N. Cho, D. Y. Kim, and Y. C. Kim, J. Appl. Phys. 93, 1367 (2003).

${ }^{9}$ S. Furumi, S. Yokoyama, A. Otomo, and S. Mashiko, Appl. Phys. Lett. 82, 16 (2003).

${ }^{10}$ S. Yokoyama, A. Otomo, and S. Mashiko, Appl. Phys. Lett. 84, 2491 (2004).

${ }^{11}$ A. Chanishvili, G. Chilaya, G. Petriashvili, R. Barberi, R. Bartolino, G. Cipparrone, A. Mazzulla, and L. Oriol, Appl. Phys. Lett. 83, 5353 (2003).

${ }^{12}$ A. Y. G. Fuh, T. H. Lin, J. H. Liu, and F. C. Wu, Opt. Express 12, 1857 (2004).

${ }^{13}$ S. M. Morris, A. D. Ford, M. N. Pivnenko, and H. J. Coles, J. Appl. Phys. 97, 023103 (2005).

${ }^{14}$ S. M. Morris, A. D. Ford, B. J. Broughton, M. N. Pivnenko, and J. J. Coles, Proc. SPIE, 5741, 118 (2005).

${ }^{15}$ F. Araoka, K. Shin, Y. Takanishi, K. Ishikawa, H. Takezio, Z. Zhu, and T. M. Swager, J. Appl. Phys. 94, 279 (2003).

${ }^{16}$ Q. Hong, T. X. Wu, and S. T. Wu, Liq. Cryst. 30, 367 (2003). 